\title{
Lipid Storage Diseases
}

National Institute of Neurological Disorders and Stroke (NINDS)

\section{Source}

National Institute of Neurological Disorders and Stroke (NINDS). Lipid Storage Diseases

Information Page.

Lipid storage diseases are a group of inherited metabolic disorders in which harmful amounts of fatty materials (lipids) accumulate in various tissues and cells in the body. Lipids are important parts of the myelin sheath that coats and protects the nerves. Over time, this excessive storage of fats can cause permanent damage to cells and tissues in the brain and peripheral nervous system, and in other parts of the body. Symptoms may appear early in life or develop in the teen or even adult years. Neurological complications of the lipid storage diseases may include:

- lack of muscle coordination,

- brain degeneration,

- seizures,

- loss of muscle tone,

- learning problems,

- spasticity,

- feeding and swallowing difficulties,

- slurred speech,

- increased sensitivity to touch,

- pain in the arms and legs, and

- clouding of the cornea. 